\title{
VACCINATION OF PATIENTS WITH DIABETES MELLITUS - A RETROSPECTIVE STUDY
}

\author{
Rastislav Mad'ar1, 2, Dagmar Benešová1, Dana Brandejská1, Miriam Čermáková1, Alena Dvořáková1,3, Olga \\ Gazárková1, Silvana Jakubalová1, Ilona Kochová1, Jana Laštovičková1,4, Dagmar Nebáznivá1, 5 , Marta Orolinová1, \\ Karel Polomis ${ }^{1}$, Václav Řehka ${ }^{1,4}$, Ludmila Sattranová1, Miriam Schejbalová1,, , Alena Slámová1, 6 , Deanna \\ Skalleová1, Hana Ševčíková1, Hana Tkadlecová1,7, Marta Tmějová1, Josef Trmal ${ }^{1,5}$, Dagmar Turková1 \\ ${ }^{1}$ Vaccination centres Avenier, Czech Republic \\ ${ }^{2}$ Comenius University, Jessenius Faculty of Medicine, Martin, Slovakia
}

${ }^{3}$ Regional Public Health Authority Jihlava, Czech Republic

${ }^{4}$ Regional Public Health Authority Brno, Czech Republic

${ }^{5}$ Regional Public Health Authority Ústí nad Labem, Czech Republic

${ }^{6}$ Charles University, First Faculty of Medicine, Prague, Czech Republic

${ }^{7}$ Regional Public Health Authority Zlín, Czech Republic

\section{SUMMARY}

402 subjects with diabetes mellitus have been vaccinated of the total of 34,000 vaccinees immunized during the study period of 9 and half months. Altogether 229 diabetic patients (56.97\%) have been vaccinated against tick-borne encephalitis (TBE) and 74 (18.4\%) against viral hepatitis (41 types $A+B, 30$ type $A, 3$ type B). The average age in four most commonly administered vaccines (FSME IMMUN 0.5 ML, Twinrix Adult, Typhim Vi, and Havrix 1440) was 65, 52, 56, and 54 years, respectively. Live attenuated vaccines have been given to 6 patients with diabetes $(1.49 \%)-5$ travellers to endemic countries received the yellow fever vaccine Stamaril ( 1 female, 4 male) and one male patient varicella vaccine Varilrix. Among the least common vaccines in diabetic patients were those against invasive pneumococcal and meningococcal infections. Not a single unexpected side effect has been observed following the vaccination procedure in any diabetic patient.

Based on the results of this retrospective study we can conclude that vaccination in diabetic patients is free of any risk - provided that there are no other contraindications, e.g. allergy to vaccine components or severe acute febrile illness. In the case of unstable glycaemia and significantly impaired immune system due to diabetes mellitus, vaccination with live attenuated vaccines should be carefully considered and measured against the risks of exposure to each and every specific infectious agent.

There is no reason to be afraid of vaccination in diabetic patients provided that general contraindications are respected. On the contrary, this risk group can benefit from vaccination more remarkably since it may have some life-saving potential.

Key words: diabetes mellitus, vaccination, killed vaccines, live attenuated vaccines, contraindications, side-effects, safety, primary care, vaccination rate, prevention

Address for correspondence: R. Mad’ar, Očkovací centrum, Poliklinika, Dr. Martínka 7, 70030 Ostrava-Hrabůvka, Czech Republic. E-mail: Rastislav.Madar@avenier.cz

\section{INTRODUCTION}

Vaccination is ranked between the greatest achievements in human history and in the medical world. Names like Jenner, Pasteur, Calmette, Salk, Sabin, Behring etc. are known to wide public. Thanks to them, humankind has managed to eradicate the deadly and mutilating smallpox and paralysing poliomyelitis is nowadays present only in a few developing countries. Fatal neonatal tetanus is becoming rare even in African countries and the incidence of fearful measles is steeply declining (1-6).

Whilst vaccination coverage of the paediatric population in the Czech Republic is one of the highest in the world reaching up to 99 per cent, utilising vaccines guaranteed by the government and provided free of charge, a different picture of the vaccination take up in the adult population is often seen to be a long way behind other developed EU countries. Some vaccines and their producers have to face fake allegations about their serious side effects or contagiousness. These are literally handed-down from generation to generation without any scientific evidence. Despite the fact that vaccination experts argue against these myths, many laymen tend to believe them and spread the false stories further. However, what is even more startling is that these fabrications sometimes find their breeding grounds among primary care physicians. They are flooded with many novelties of the medical world from different specialities and often fail to recognise those that are of an important origin, scientific value and significance. Many primary care physicians for adults only vaccinate their patients against tetanus and a few patients against influenza and are simultaneously ignoring a wide range of other vaccines with life saving as well as cost saving potential. Some GPs, for example, refuse to vaccinate against flu from January onwards or against tick-borne encephalitis in the summer months, which 
is in both cases medically unjustifiable. Many are also not willing to offer vaccination to individuals with chronic long-lasting health problems, even if there are no real contraindications. There appears to be a lack of GP understanding in that, inactivated (killed) vaccines are prepared and designed primarily for elderly individuals and persons with various medical impairments e.g. cardio-vascular or respiratory diseases, diabetes mellitus, renal or immunity impairment, etc.

Immunisation saves lives and also money both to a vaccinated individual and health insurance companies, consequently to, the state budget. Many diseases leave infected people permanently disabled - paralysed, deaf or having mental problems. They may markedly lose their quality of life even if its longevity is not reduced. Vaccination is undoubtedly the foundation stone of the future of medicine and plays very important role irrespective of medical speciality. Increasing the vaccination coverage of the population is the part to be played especially by primary care specialists. Specialised vaccination centres play a complementary role and are only secondary to GPs. Therefore it is very important that all general practitioners are forthcoming with truthful evidence based information and accurate data about all vaccines available to each individual in the respective country. The scientific, proven and practically verified data needs to be provided by vaccination specialists and presented to GPs who often lack time to study topical information from each and every branch of medicine.

The Avenier network of vaccination centres is the largest of its kind in the Czech Republic. Currently, it consists of 19 outpatient vaccination clinics all over the country serving more than 60 thousand clients each year. Their unique practical experience and use of flexible software for collecting and storing patient information enables us to perform retrospective data analysis of any risk factors in vaccinated individuals and share this valuable information with general practitioners, as well as, with other members of medical community.

\section{METHODS}

By the use of the Avenier database it has been possible to perform a retrospective analysis of vaccines administered to patients with diabetes mellitus in the period of 9 and a half months from the 1st of October 2009 till the 15th of July 2010. Data has been inserted into MS Navision programme by vaccination centres (VC) physicians and subsequently transferred to MS Excell. Taking a medical history of each vaccinated individual is a routine part in every vaccination procedure. Before the vaccination is considered, every physician should enquire data about acute and chronic illnesses, vaccination history, allergies, current and recent medical treatment as well as other important issues of any relevance with respect to planned vaccination. These categories have to be filled in the MS Navision programme even if no risk factors are present stating NEGATIVE. The medication of diabetic patients (insulin and peroral antidiabetics) has been followed up during the last 2 and a half months of the study. MS Navision data do not differentiate between DM type 1 and type 2, however, serious immune system impairment due to unstable disease would be recorded.

In total 20 vaccination centres (of Avenier) took part in a study located all over the country in the cities of Prague (3 VC), Ostrava
(2 VC), České Budějovice (2 VC), Plzeň, Olomouc, Zlín, Brno, Břeclav, Znojmo, Jihlava, Plzeň, Hradec Králové, Liberec, Ústí nad Labem, Karlovy Vary and Sokolov - each with 1 vaccination centre. The 20th vaccination centre in Sokolov ceased its activity at the end of June 2010.

\section{RESULTS}

During the study period of 9 and a half months, 402 vaccines $(1.18 \%)$ have been administered to patients with diabetes mellitus out of 34,055 vaccines administered in total to all vaccinated subjects.

Altogether 229 diabetic patients (56.97\%) have been vaccinated against tick-borne encephalitis (TBE) and 74 (18.4\%) against viral hepatitis (41 types $\mathrm{A}+\mathrm{B}, 30$ type $\mathrm{A}, 3$ type $\mathrm{B}$ ).

The most commonly administered vaccine to patients with this chronic condition was the one against tick-borne encephalitis FSME-IMMUN 0.5 ML used in 223 cases (55.47\%; 91 female and 132 male), followed by Twinrix Adult in 41 (10.20\%; 13 female and 28 male), Typhim Vi in $26(6.47 \%$; 9 female and 17 male), and Havrix 1440 in 24 cases $(5.97 \%$; 9 female and 15 male) (Table $1)$. The average age in these four most commonly administered vaccines was $65,52,56$, and 54 years, respectively. The use of insulin had been documented in 73 vaccinated individuals, out of which 44 have received vaccine FSME-IMMUN 0.5 ML (60.27\%; average age 60 years), followed by Silgard in 8 (10.96\%; average

Table 1. Vaccines administered to patients with diabetes mellitus

\begin{tabular}{|l|c|}
\hline Vaccine & Number of applications \\
\hline AVAXIM 160 & 6 \\
\hline CERVARIX & 1 \\
\hline DUKORAL & 2 \\
\hline ENCEPUR for adults & 6 \\
\hline ENGERIX-B & 3 \\
\hline FLUAD & 2 \\
\hline FSME-IMMUN 0.5 ML & 223 \\
\hline HAVRIX 1440 & 24 \\
\hline INFLUVAC & 19 \\
\hline MENINGOCOCCALA+C & 2 \\
\hline PNEUMO 23 & 6 \\
\hline SILGARD & 8 \\
\hline STAMARIL & 5 \\
\hline TETAVAX & 2 \\
\hline TWINRIX ADULT & 41 \\
\hline TYPHERIX & 9 \\
\hline TYPHIM VI & 26 \\
\hline VARILRIX & 1 \\
\hline VAXIGRIP & 15 \\
\hline VERORAB & 1 \\
\hline TOTAL & 402 \\
\hline
\end{tabular}


age 16 years) and Twinrix Adult in 5 patients (6.85\%; average age 46 years). Out of 165 patients using peroral antidiabetics, 105 have been vaccinated by FSME IMMUN 0.5 ML (63.63\%; average age 65 years), followed by Twinrix Adult in 17 (10.30\%; average age 55 years), Typhim Vi in 13 (7.88\%; average age 58 years) and Havrix 1440 in 11 patients (6.67\%; average age 57 years). Only 1 diabetic patient vaccinated against viral hepatitis of any type was younger than 20 years of age.

The distribution of the most commonly administered FSME IMMUN vaccine according to age group is seen in Fig. 1. An overwhelming majority of all individuals vaccinated by FSME IMMUN consisted of the elderly over 60 years of age (164; $73.54 \%$ ). Only one vaccinated subject with diabetes has been younger than 19 years of age. Not a single FSME IMMUN 0.25 ML nor Encepur for children vaccines intended for minors up to 16 (resp. 12) years of age has been administered to diabetic patients.

Influenza vaccines of any type were administered to only 36 diabetic patients $(8.95 \%)$, none of them was younger than 30 years of age.

Notably, one of the least common vaccines is the 23-valent polysaccharide pneumococcal vaccine (PPV) Pneumo 23 that has been given only to 6 individuals with diabetes $(1.49 \%)-5$ of them aged 60-69 years and 1 aged between 50-59 years of age.

Polysaccharide meningococcal vaccine $\mathrm{A}+\mathrm{C}$ has been given only to 2 subjects with diabetes $(0.5 \%)$ aged 48 and 58 . Conjugated meningococcal vaccine has not been administered at all - not even to one subject with diagnosis diabetes mellitus.

Live attenuated vaccines have been given to 6 patients with diabetes $(1.49 \%)-5$ travellers to endemic countries received the yellow fever vaccine Stamaril (1 female, 4 male) and one male patient was vaccinated by varicella vaccine Varilrix.

Not a single unexpected side effect has been observed following use of any vaccine in any diabetic patient.

\section{DISCUSSION}

Based on the results of this retrospective study we can conclude that vaccination in diabetic patients is free of any risk - provided that there are no other contraindications, e.g. allergy to vaccine

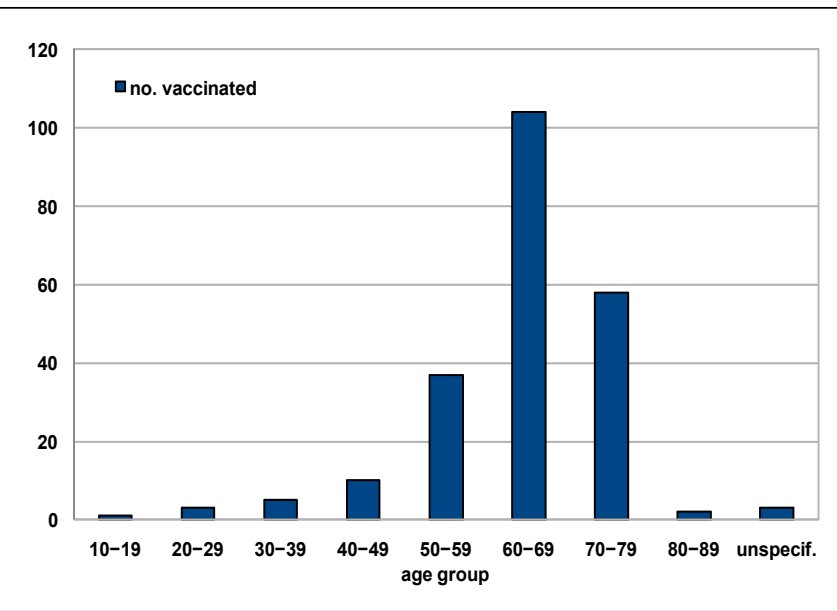

Fig. 1. The distribution of FSME IMMUN vaccine according to age groups. components or severe acute febrile illness. In the case of unstable glycaemia and significantly impaired immune system due to diabetes mellitus, vaccination with live attenuated vaccines should be carefully considered and measured against the risks of exposure to each and every specific infectious agent.

The vaccine against tick-borne encephalitis (FSME IMMUN $0.5 \mathrm{ML}$ ) for adults and children from 16 years of age has been used most commonly in diabetic patients. This very likely reflects the deteriorating epidemiological situation in the Czech Republic with more than 800 cases of TBE reported last year and enlargement of endemic areas and emergence of new ones all over the country. Most people interested in this kind of vaccination change their mind based on information published in the mass media. The majority of individuals vaccinated against TBE were elderly and over 60 years of age.

The second most common vaccines used in diabetic patients are those against viral hepatitis $(\mathrm{A}+\mathrm{B}$ or $\mathrm{A})$. Awareness about this kind of vaccine in the lay population may come from the all-country outbreak of hepatitis A in 2008 with hundreds of cases being given high media attention. However, only one diabetic patient younger than 20 years of age has been vaccinated against viral hepatitis and not a single pediatric TBE vaccine has been given to a child suffering from diabetes mellitus.

Ultimately more male diabetic patients were vaccinated than female.

Very low numbers of patients have been vaccinated with 23-valent PPV, as well as, by both types of meningococcal vaccines. The need for and desirabilty of pneumococcal vaccination in the elderly and in individuals with chronic conditions is severly underestimated and it is therefore underused. Increased vaccination coverage in the main target groups would not only be significantly cost-effective but would also notably reduce morbidity and mortality. There is also great potential for improvement in the uptake of vaccination against influenza.

The application of a vaccine stimulates the human immune system at various levels. It activates the natural immune processes and does not present any extraordinary burden. By means of simulation of natural infection through the application of safe antigen it stimulates the humoral and/or cellular immunity.

Vaccines nowadays have to undergo 4 phases of strict clinical trials supervised by top experts and subsequently assessed by neutral non-commercial opinion leaders. Advances in medicine and new technologies bring vaccines of a better quality. At the same time, vaccines against new diseases are under development. Specialists from the Avenier vaccination centres lead the way in the application of all registered vaccines. In the overwhelming majority there are no side effects whatsoever, not even local ones in the site of injection. Serious unexpected side effects are so rare that most vaccinating physicians do not experience any such event during their entire professional careers. By far the greater risks to human health are to be found in common everyday activities such as driving or house work.

Primary care physicians should target their preventive efforts, including vaccination, especially at individuals under the risk of serious diseases and their sequelae. In case they do not feel confident in the vaccination of individuals with impaired health of any origin they can with confidence refer their patients to specialists in vaccination centres. Promoting methods of primary disease prevention and encouraging patients to take advantage 
of vaccination programmes is much needed in order to protect those at highest risk.

\section{CONCLUSION}

This retrospective study shows that the vaccination of patients suffering from diabetes mellitus does not pose increased risk of serious side effects. There is no reason to be afraid of vaccination in diabetic patients provided that general contraindications are respected. On the contrary, this risk group can benefit from vaccination more remarkably since it may have some life-saving potential. In agreement with other authors (7-10) we conclude that the vaccination of people with chronic conditions including diabetes mellitus should be an indispensable part of the preventive care in every country including the Czech Republic.

This study was financially supported by the Avenier Inc.

\section{REFERENCES}

1. Mad'ar R, et al. How to increase vaccination rate in our population, especially in adults. Očkování a cestovní medicína. 2010;1(1):1-2. (In Czech.)
2. Blencowe H, Lawn J, Vandelaer J, Roper M, Cousens S. Tetanus toxoid immunization to reduce mortality from neonatal tetanus. Int J Epidemiol. 2010 Apr;39 Suppl 1:i102-9.

3. Centers for Disease Control and Prevention (CDC). Progress toward poliomyelitis eradication - Nigeria, January 2009-June 2010. MMWR Morb Mortal Wkly Rep. 2010 Jul 9;59(26):802-7.

4. Leuridan E, Hens N, Hutse V, Ieven M, Aerts M, Van Damme P. Early waning of maternal measles antibodies in era of measles elimination: longitudinal study. BMJ. 2010 May 18;340:c1626.

5. Modlin JF. The bumpy road to polio eradication. N Engl J Med. 2010 Jun 24;362(25):2346-9.

6. Validation of neonatal tetanus elimination in Gujarat and Himachal Pradesh, India, 2008. Wkly Epidemiol Rec. 2010 Jul 2;85(27):265-71.

7. Giannattasio A, Squeglia V, Lo Vecchio A, Russo MT, Barbarino A, Carlomagno R, et al. Pneumococcal and influenza vaccination rates and their determinants in children with chronic medical conditions. Ital J Pediatr. 2010 Mar 26;36:28.

8. Gutiérrez G, Pérez-Cuevas R, Levy S, Reyes H, Acosta B, Cantón SF, et al. Strengthening preventive care programs: a permanent challenge for healthcare systems; lessons from PREVENIMSS México. BMC Public Health. 2010 Jul 14;10:417.

9. Richardson DK, Helderman T, Lovett P. Meningococcal epiglottitis in a diabetic adult patient: a case report. J Emerg Med. In press.

10. Rodríguez-Rieiro C, Domínguez-Berjón MF, Esteban-Vasallo MD, Sánchez-Perruca L, Astray-Mochales J, Fornies DI, et al. Vaccination coverage against 2009 seasonal influenza in chronically ill children and adults: analysis of population registries in primary care in Madrid (Spain). Vaccine. 2010 Aug 31;28(38):6203-9.

Received August 10, 2010 Accepted in revised form March 2, 2011 